\title{
Superposed Output Coherent and Squeezed Laser Light Beams
}

\author{
Habtamu Dagnaw \\ Department of Physics, Injibara University, PO box 040, Injibara, Ethiopia
}

\begin{abstract}
The aim of this paper is to analyze the squeezing and statistical properties of superposed output coherent and squeezed laser light beams. In order to carry out the analysis, we have obtained the superposition density operator along with the $\mathrm{Q}$ functions, we have calculated the mean photon number, the photon number variance and the quadrature variances for the superposed output light beams. It has been found that the mean photon number and quadrature variances of the superposed output light beams are the sum of the mean photon number and quadrature variances of the separate light beams, respectively. In addition, the mean photon number and quadrature squeezing of the superposed output light beams increase with linear gain coefficient.
\end{abstract}

Keywords: Superposed, Squeezing, Density operator, Coherent light, Photon statistics.

DOI: $10.7176 / \mathrm{APTA} / 77-03$

Publication date:May $31^{\text {st }} 2019$

\section{Introduction}

In quantum optics, the annihilation and creation operator describing a single-mode radiation can be decomposed into two component operators, referred to as quadrature operators [1]. In a squeezed state the quantum noise in one quadrature is below the vacuum level at the expense of enhanced fluctuations in the conjugate quadrature, with the product of the uncertainties in the two quadratures satisfying the uncertainty relation. Squeezing like photon antibunching or sub-Poissonian photon statistics is a nonclassical feature of light. Squeezed light has potential applications in the detection of weak signals and in low-noise communications [2-6].

A three-level laser is quantum optical system in which three level atoms in a cascade configuration, initially prepared in a coherent superposition of the top and bottom levels, are injected into a cavity coupled to a vacuum reservoir via a single-port mirror. When a three-level atom in cascade configuration, it makes a transition from the top to the bottom level via the intermediate level, two photons are generated. If the generated light modes have the same frequency, the three-level laser is said to be a degenerate three-level laser; otherwise it is called a nondegenerate three-level laser $[2,7]$.

Some authors have arrived at the conclusion that the superposition of coherent light beam with some other light beam does not affect the quadrature variance of the other light beam $[8,9]$. Fesseha has studied the Statistical and Squeezing properties of superposed coherent and squeezed light produced by one mode Subharmonic generations in the same cavity. Applying a slightly modified definition for the quadrature Variance of a pair of superposed light beams and comparing with the quadrature variance of a single coherent light beam. He has shown the quadrature squeezing of the superposed light beams is half of the squeezed light. This is just the average quadrature squeezing of the separate light beams [10]. Moreover, Habtamu has studied the statistical and squeezing properties of superposed three light beams. He carried out the analysis applying the superposition density operator along with the Q-functions. He has shown the quadrature squeezing of the superposed three light beams is the average quadrature squeezing of the three light beams. In addition, the presence of the coherent light decreases the quadrature squeezing of the superposed three light beams [11].

In this paper, we seek to analyze the squeezing and statistical properties of superposed output coherent and squeezed laser light beams produced by a coherent light and degenerate three-level laser. We arrange our system in such a way that the output light (LB1) from the laser is incident on a side of a perfectly transmitting mirror(M) while the output light beam (LB2) from Coherent light is incident on a side of perfectly reflecting mirror (M) as depicted in the figure 1 . In order to carry out the analysis, we first obtain the density operator for the superposed light beams in terms of the respective Q-functions. 


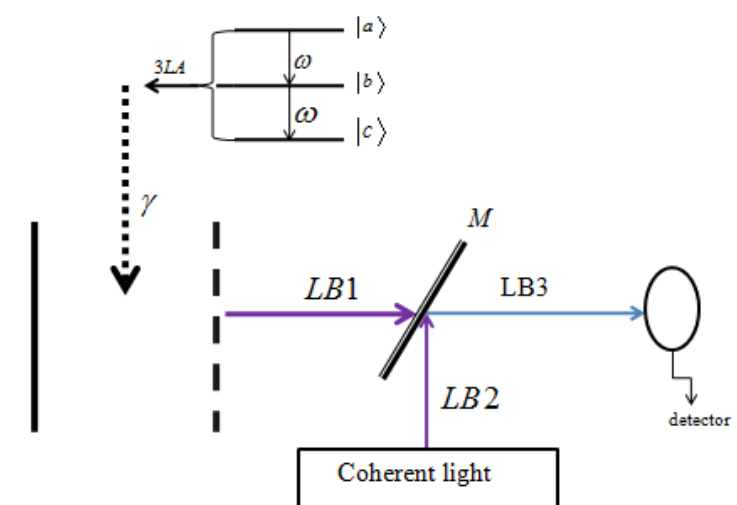

Figure 1 Schematic Design for the system.

\section{Density Operator}

Here we seek to determine the density operator for superposed light beams. Suppose $\hat{\rho}^{\prime}\left(\right.$ 浲 $\left.a_{1}, t\right)$ is the density operator for the first light beam, say for the laser light beam. Then upon expanding this density operator in normal order and applying the completeness relation for coherent state, we see that

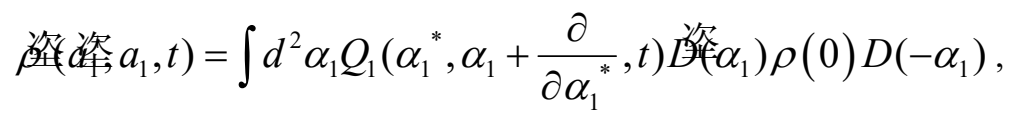

where

$$
Q_{1}\left(\alpha_{1}{ }^{*}, \alpha_{1}+\frac{\partial}{\partial \alpha_{1}{ }^{*}}, t\right)=\frac{1}{\pi} \sum_{i j} C_{i j} \alpha_{1}{ }^{{ }^{i} i}\left(\alpha_{1}+\frac{\partial}{\partial \alpha_{1}{ }^{*}}\right)^{j} \text {, }
$$

is the Q-function associated with the laser light beam and $\hat{\rho}(0)=|0\rangle\langle 0|$ represents the density operator at initial time which is vacuum state.

On the bases of Eq. (1), the density operator for superposition of the second light beam with the first one can be written as

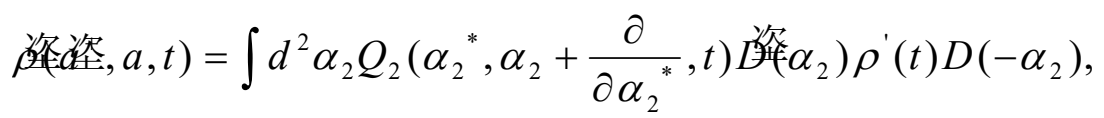

in which

$$
Q_{2}\left(\alpha_{2}{ }^{*}, \alpha_{2}+\frac{\partial}{\partial \alpha_{2}{ }^{*}}, t\right)=\frac{1}{\pi} \sum_{k l} C_{k l} \alpha_{2}^{* k}\left(\alpha_{2}+\frac{\partial}{\partial \alpha_{2}{ }^{*}}\right)^{l},
$$

is the Q-function associated with the coherent light beam. Now by combination of Eqs. (1) and (3) then using Baker-Hausdorff identity and the definition that the coherent state is the displacement operator acting on a vacuum state, we easily obtain

$$
\hat{\rho}(\text { 深, } a, t)=\int d^{2} \alpha_{1} d^{2} \alpha_{2} Q_{1}\left(\alpha_{1}{ }^{*}, \alpha_{1}+\frac{\partial}{\partial \alpha_{1}{ }^{*}}, t\right) Q_{2}\left(\alpha_{2}{ }^{*}, \alpha_{2}+\frac{\partial}{\partial \alpha_{2}{ }^{*}}, t\right)\left|\alpha_{1}+\alpha_{2}\right\rangle\left\langle\alpha_{2}+\alpha_{1}\right| \text {. }
$$

Eq. (5) represents the density operator of light beams (LB3) as shown in figure 1.

\section{Photon Statistics}

In this section, we calculate the mean and variance of the photon number for the superposed output light beams employing the resulting density operator and the Q-functions of the respective light beams.

\subsection{The Mean Photon Number}

The mean photon number of the superposed output light beams can be expressed in terms of the density operator as

$$
\bar{n}_{\text {out }}=\operatorname{Tr}\left(\hat{\rho}(t) \text { 㫊 } a_{\text {out }}\right) \text {, }
$$

Employing the input-output relation

$$
\text { 疼 }{ }_{\text {ut }}=\sqrt{k} a-a_{i n} \text {, }
$$

where ' $k$ ' is the cavity damping constant and $\hat{a}_{i n}$ annihilation operator for cavity mode light. 
稘 $a_{1 \text { out }}+a_{2 \text { out }}$, is the annihilation operator for the superposed output light beams.

The system is coupled to vacuum reservoir, Eq. (7) can be written as

疼 $u$, $=\sqrt{k} a$.

Applying Eq. (5) and Eq. (8) in Eq.(6), we get

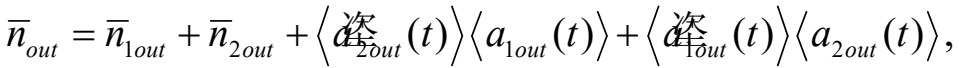

in which

$\left\langle\hat{a}_{\text {iout }}(t)\right\rangle=\int d^{2} \alpha_{i} Q_{i}\left(\alpha_{i}^{*}, \alpha_{i}+\frac{\partial}{\partial \alpha_{i}^{*}}, t\right) \alpha_{i}, i=1,2$.

We next proceed to determine the expectation value $\hat{a}_{\text {lout }}(t)$, we can write as

$\left\langle\hat{a}_{1 \text { out }}(t)\right\rangle=\int d^{2} \alpha_{1} Q_{1}\left(\alpha_{1}^{*}, \alpha_{1}+\frac{\partial}{\partial \alpha_{1}^{*}}, t\right) \alpha_{1}$,

in which

$Q_{1}\left(\alpha_{1}^{*}, \alpha_{1}+\frac{\partial}{\partial \alpha_{1}^{*}}, t\right)=Q_{1}\left(\alpha_{1}^{*}, \alpha_{1}, t\right) \exp \left[\left(v_{1} \alpha_{1}-u_{1} \alpha_{1}^{*}\right) \frac{\partial}{\partial \alpha_{1}{ }^{*}}+\frac{v_{1}}{2} \frac{\partial^{2}}{\partial \alpha_{1}^{* 2}}\right]$,

Where

$Q_{1}\left(\alpha_{1}, \alpha_{1}^{*}, t\right)=\frac{\sqrt{u_{1}^{2}-v_{1}^{2}}}{\pi} \exp \left[-u_{1} \alpha_{1}^{*} \alpha_{1}+v_{1}\left(\alpha_{1}^{2}+\alpha_{1}^{* 2}\right) / 2\right]$,

With

$u_{1}=\frac{a_{1}}{a_{1}^{2}-b_{1}^{2}}, v_{1}=\frac{b_{1}}{a_{1}^{2}-b_{1}^{2}}$,

$a_{1}=1+\frac{A_{1}(1-\eta)}{2\left(A_{1} \eta+k_{1}\right)}\left(1-e^{-\left(A_{1} \eta+k_{1}\right) t}\right.$,

$b_{1}=\frac{A_{1} \sqrt{1-\eta^{2}}}{2\left(A_{1} \eta+k_{1}\right)}\left(1-e^{-\left(A_{1} \eta+k_{1}\right) t}\right.$.

Using Eq. (12) in Eq.(11), we can be put in the form

$$
\left\langle\hat{a}_{1 \text { out }}(t)\right\rangle=\left.\frac{\partial}{\partial \lambda} \int d^{2} \alpha_{1}\left[Q_{1}\left(\alpha_{1}^{*}, \alpha_{1}, t\right) D\left(\lambda, \alpha^{*}, \alpha_{1}\right)\right]\right|_{\lambda=0},
$$

Where

$D\left(\lambda, \alpha^{*}{ }_{1}, \alpha_{1}\right)=\exp \left[\left(v_{1} \alpha_{1}-u_{1} \alpha^{*}{ }_{1}\right) \frac{\partial}{\partial \alpha_{1}^{*}}+\frac{v_{1}}{2} \frac{\partial^{2}}{\partial \alpha_{1}^{* 2}}\right] \exp \left(\lambda \alpha_{1}\right)$.

Based on eigenvalue equation for a differential operator $\hat{\mathrm{A}}[11]$. We easily get

$D\left(\lambda, \alpha^{*}, \alpha_{1}\right)=\exp \left(\lambda \alpha_{1}\right)$.

Substituting Eqs. (19) and (13) into Eq.(17), then carrying out the integration and upon performing the differentiation and also setting $\lambda=0$, we get $\left\langle\hat{a}_{1 \text { out }}(t)\right\rangle=0$.

Following a similar procedure, one can easily verify that .

$\left\langle\hat{a}_{1 \text { out }}^{+}(t)\right\rangle=0$,

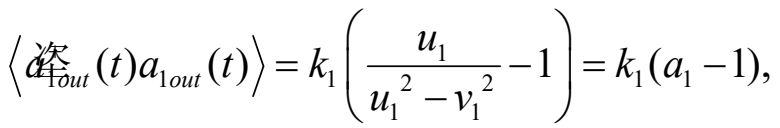

$\left\langle\right.$ 疼。ut $\left.(t) a_{2 \text { out }}(t)\right\rangle=k_{2} q^{2}$, 
$\left\langle\right.$ 路out $\left.a_{1 \text { out }}^{2}\right\rangle=2 \bar{n}_{1 \text { out }}^{2}+k_{1}^{2} b_{1}^{2}$,

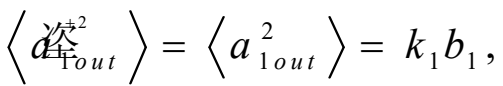

$\left\langle\right.$ 篹 $\left.a^{2}\right\rangle=2 \bar{n}_{1}^{2}+\left(k_{1} b_{1}\right)^{2}+\bar{n}_{2}^{2}+2 \bar{n}_{2} k_{1} b_{1}+4 \bar{n}_{1} \bar{n}_{2}$,

and

$\left\langle\hat{a}_{2 \text { out }}(t)\right\rangle=\sqrt{k_{2}} q$,

in which

$q=\frac{2 \varepsilon}{k_{2}}\left(1-e^{\frac{-k_{2} t}{2}}\right)$.

Finally, the mean photon number of the superposed output light beams is

$\bar{n}_{s}=\frac{4 \varepsilon^{2}}{k_{2}}\left(1-e^{-k_{2} t / 2}\right)^{2}+\frac{A_{1} k_{1}(1-\eta)}{2\left(A_{1} \eta+k_{1}\right)}\left(1-e^{-\left(A_{1} \eta+k_{1}\right) t}\right)$.

At steady state the mean photon number turns out to be

$\bar{n}_{s}=\frac{4 \varepsilon^{2}}{k_{2}}+\frac{A_{1} k_{1}(1-\eta)}{2\left(A_{1} \eta+k_{1}\right)}$.

We observe from Eq. (30) that the mean photon number of the superposed output light beams is the sum of the mean photon numbers of output coherent light and degenerate three-level laser.

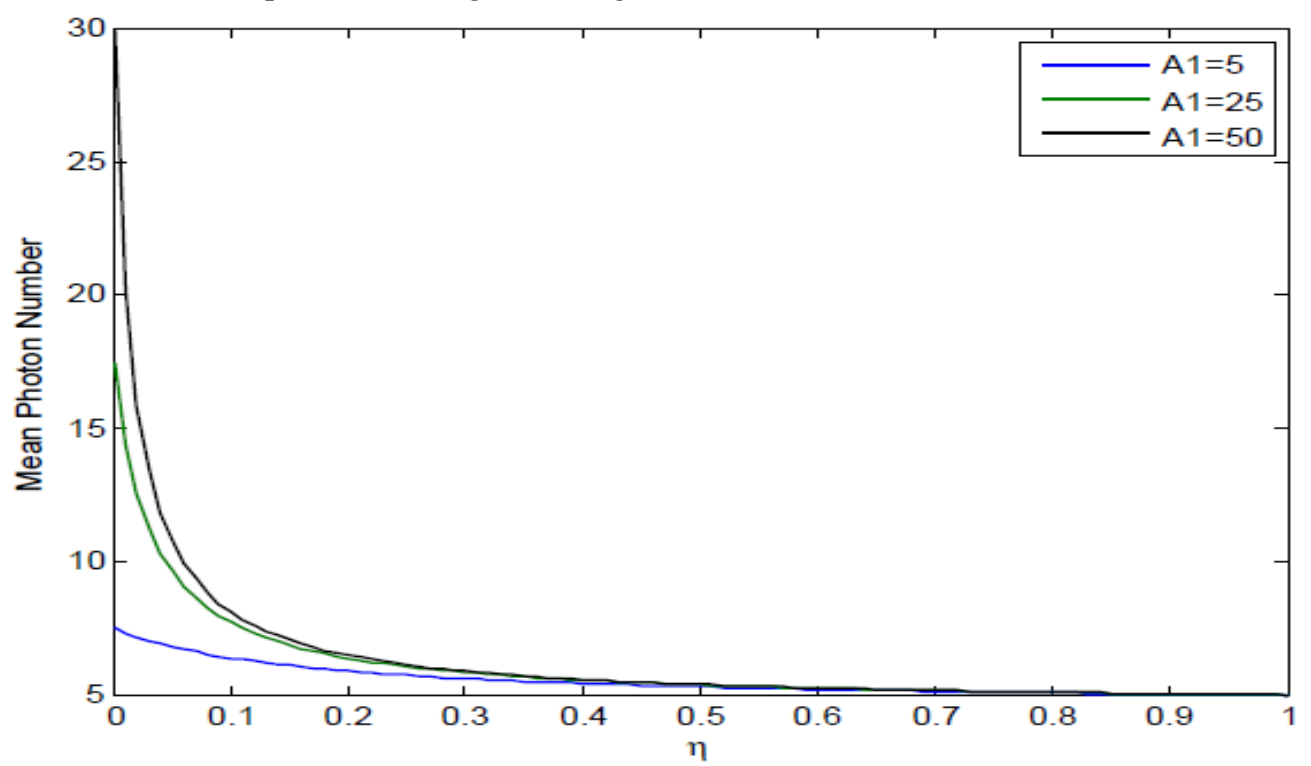

Fig. 2: Plots of the mean photon number [Eq. (30)] versus $\eta$ for $\mathrm{k}_{1}=\mathrm{k}_{2}=0.8, \varepsilon=1$, and for different values liner gain coefficients $\left(\mathrm{A}_{1}\right)$. The figure shows that the mean photon number increases with the liner gain coefficients $\left(\mathrm{A}_{1}\right)$ and decreases with $\eta$. 


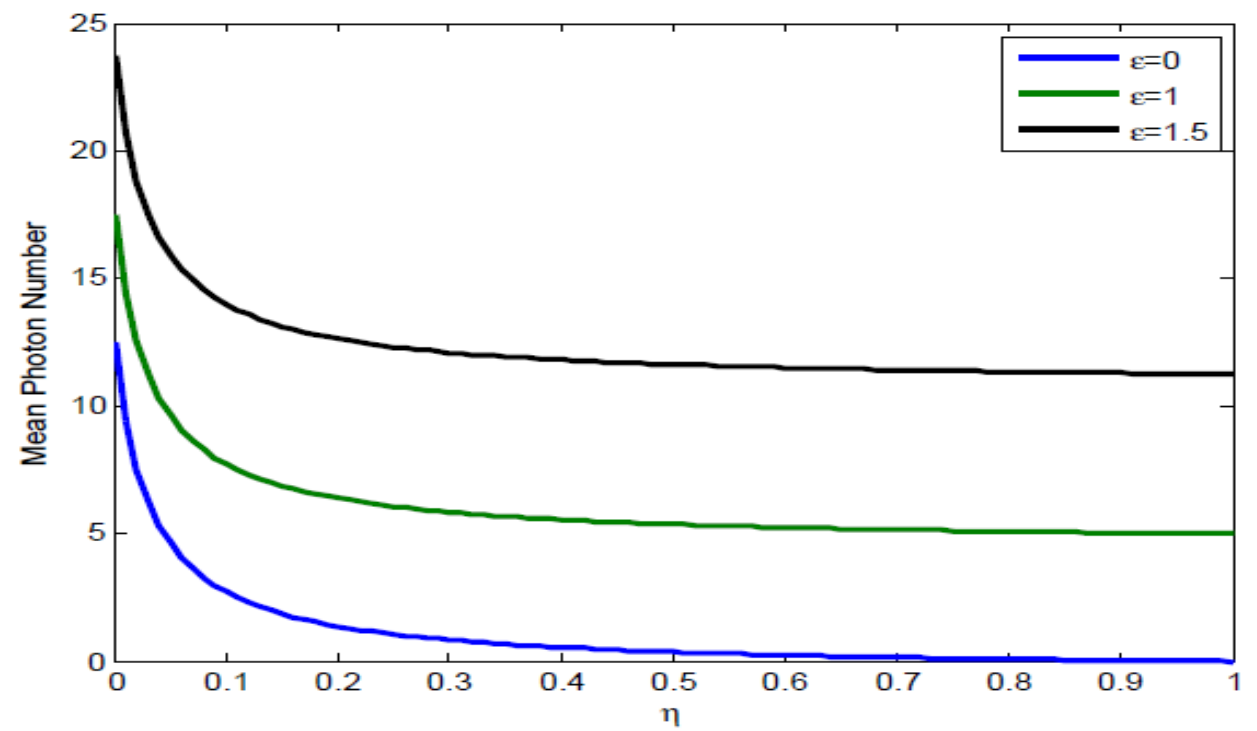

Fig.3: Plots of the mean photon number [Eq. (30)] versus $\eta$ for $\mathrm{k}_{1}=\mathrm{k}_{2}=0.8, \mathrm{~A}_{1}=25$, and for different values of $\varepsilon$.

The figure indicates that the mean photon number increases with $\varepsilon$ and decreases with $\eta$.

\subsection{The Variance of Photon Number}

The variance of the photon number for the superposed output light beams is defined by

$$
\Delta n_{s}^{2}=\left\langle(\text { 淕 }(t) a(t))^{2}\right\rangle-\bar{n}_{s}^{2} \text {. }
$$

Using commutation relation

$$
\left[\text { 次 } a^{+}\right]=k_{1}+k_{2} \text {, }
$$

with the aid of Eq. (29), Eq. (28) can be written in the form

$$
\Delta n_{s}^{2}=\left\langle\text { 燃 }^{2} a^{2}\right\rangle-\bar{n}_{s}^{2}+\left(k_{1}+k_{2}\right) \bar{n}_{s} \text {. }
$$

On account of eq. (26), the variance of the photon number for the superposed output light beams turns out to

$\Delta n_{s}^{2}=2 \bar{n}_{1}^{2}+\bar{n}_{2}^{2}+\left(k_{1} b_{1}\right)^{2}+2 \bar{n}_{2} k_{1} b_{1}+4 \bar{n}_{1} \bar{n}_{2}-\bar{n}_{s}^{2}+\left(k_{1}+k_{2}\right) \bar{n}_{s}$.

We consider two special case, first case $\varepsilon=0$, Eq.(34) reduced to

$\Delta n_{s}^{2}=k_{1} \Delta n_{1 \text { out }}^{2}$.

Eq. (35) represents the variance of the photon number for the output laser light beam.

And other case, upon setting $\mathrm{A}_{1}=0$, the variance of the photon number is

$\Delta n_{s}^{2}=k_{2} \Delta n_{2 o u t}^{2}=k_{2} \bar{n}_{2 o u t}$,

Eq. (36) is the variance of the photon number for the output coherent light beam.

We easily see from Eq. (34) that the variance of photon number for superposed output light beams is greater than the mean photon number superposed output light beams. This shows that the photon statistics of the superposed output light beams is super Poissonian. The variance of photon number for superposed output light beams is does not the sum of the separate light beams.

\section{QuadratureVariance}

Here we seek to determine the quadrature variance of the superposed output light beams. The quadrature variances for the superposed output light beams can be written as

$$
(\Delta \text { 深 })_{\text {out }}=\left(\Delta a_{ \pm}^{2}\right)_{1}^{\text {out }}+\left(\Delta d_{ \pm}^{2}\right)_{2}^{\text {out }} \text {. }
$$

Since quadrature variances of output coherent light is

$$
\left(\Delta \hat{a}_{ \pm}^{2}\right)_{2}^{\text {out }}=k_{2} \text {. }
$$

Finally, the quadrature variance of the superposed output light beams is turns to be 


$$
\left(\Delta \hat{a}_{ \pm}^{2}\right)_{\text {out }}=k_{1}+k_{2}+\frac{A_{1} k_{1}\left[(1-\eta) \pm \sqrt{1-\eta^{2}}\right]}{A_{1} \eta+k_{1}}\left(1-e^{-\left(A_{1} \eta+k_{1}\right) t}\right) .
$$

We notice that for $t=0$, the quadrature variance Eq. (39) reduces to

$$
\left(\Delta \hat{a}_{ \pm}^{2}\right)_{\text {out }}=k_{1}+k_{2} \text {. }
$$

Eq. (40) represents the output quadrature variances of a pair of superposed vacuum states.

The quadrature variances at steady state found to be

$$
\left(\Delta \hat{a}_{ \pm}^{2}\right)_{\text {out }}=k_{1}+k_{2}+\frac{A_{1} k_{1}\left[(1-\eta) \pm\left(1-\eta^{2}\right)^{\frac{1}{2}}\right]}{A_{1} \eta+k_{1}} .
$$

We observe that the quadrature variance for superposed output light beams is the sum of the quadrature variances of output lights from the laser and coherent light beams.

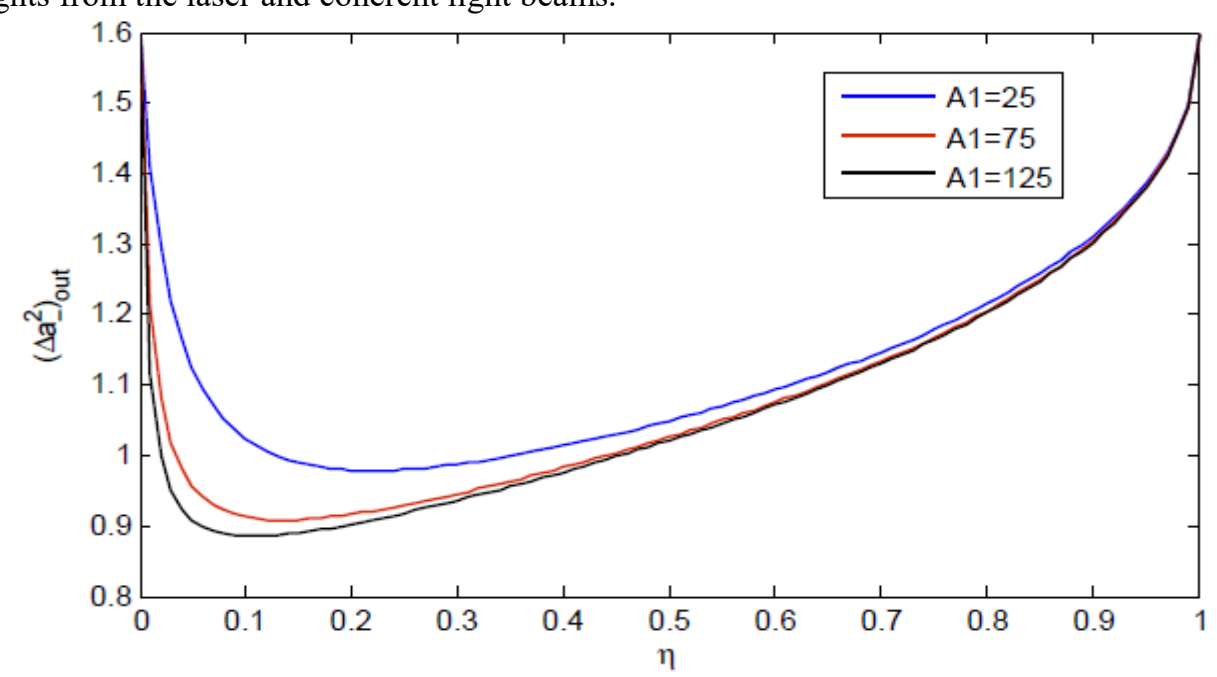

Fig.4: Plots of the quadrature variance $\left(\Delta \hat{a}_{-}^{2}\right)_{\text {out }}$ [Eq.(41) ]versus $\eta$ for $\mathrm{k}_{1}=\mathrm{k}_{2}=0.8$ and for different values of the linear gain coefficients $\left(\mathrm{A}_{1}\right)$.

Fig.4 represents the variance of the minus quadrature Eq. (41) versus $\eta$, for $\mathrm{k}_{1}=\mathrm{k}_{2}=0.8$ and for $\mathrm{A}_{1}=25, \mathrm{~A}_{1}=75$, and $A_{1}=125$. We observe from the figure the quadrature squeezing increases with the linear gain coefficients. Moreover, the maximum quadrature squeezing described by Eq. (41) for $\mathrm{A}_{1}=125$ and $\mathrm{k}_{1}=\mathrm{k}_{2}=0.8$, is found to be $44.6 \%$ and occurs at $\eta=0.11$ below the coherent state level.

\section{Conclusion}

In this paper, we have analyzed the squeezing and statistical properties of the superposed output coherent and squeezed laser light beams. In order to carry out the analysis, we have obtained the superposition density operator along with the $\mathrm{Q}$ functions, we have calculated the mean photon number, variance of the photon number, and the quadrature variance for the superposed output light beams. It has found that the mean photon number of superposed output light beams is the sum of the mean photon number of the separate light beams and quadrature variances of superposed light beams is the sum of quadrature variances of the separate light beams.

However, the photon number variance of superposed output light beams does not happen to the sum of the photon number variance of the separate light beams. And superposed output light exhibits super-Poissonian photon statistics. In addition, the mean photon number of the superposed output light beams increases with linear gain coefficient and proportional to amplitude of driving coherent light. The result shows that the presence of the coherent light decreases the quadrature squeezing of the superposed output light beams. Moreover, for $A_{1}=125$, $\mathrm{k}_{1}=\mathrm{k}_{2}=0.8$, the maximum quadrature squeezing for superposed output light beams is found to be $44.6 \%$ and occurs at $\eta=0.11$ below the coherent state level.

\section{References}

[1] D.F.Walls.(1983). Nature 306,141.

[2] Kassahun F. (2008). Fundamentals of Quantum Optics, Lulu, North Carolina. 
[3] Sculy, M. O. and Zubairy, M. S. (1997). Quantum Optics, Cambridge University Press, Cambridge.

[4] Fox, M. (2006). Quantum Optics An Introduction, Oxford University press.

[5] D. F. Walls and Milburn, G. J. (1994). Quantum Optics, Springer-Verlag, Berlin.

[6] Barnett, S. M. and Radmore, P. M. (1997). Methods in Theoretical Quantum Optics, Oxford University Press,New York.

[7] Alebachew, E. (2007). Degenerate three-level cascade laser with the cavity mode driven by coherent light, Opt. Commun. 273, 488-495.

[8] Y. Darge, T. and Kassahun, F. (2010). Coherently driven degenerate three-level laser with parametric amplifier, PMC Physics B 1.

[9] Getahun, M. (2009). Three-Level Laser Dynamics with Coherent and Squeezed Light, PhD Dissertation (Addis Ababa University)

[10] Kassahun, F. (2012). Superposed coherent and squeezed light, arXiv: 1201, 3712v1 [quant-phys].

[11] Dagnaw, H.(2018). Superposed coherent and two lasers light beams. Advances in physics Theories and Application, Vol. 73, 6-11. 